\title{
Targeting the ESCRT-III component CHMP2A for noncanonical Caspase-8 activation on autophagosomal membranes
}

\author{
Tatsuya Hattori ${ }^{1}$ - Yoshinori Takahashi $\mathbb{1}^{1} \cdot$ Longgui Chen $^{1} \cdot$ Zhenyuan Tang $^{2} \cdot$ Carson A. Wills $\mathbb{D}^{1} \cdot$ Xinwen Liang ${ }^{1}$ • \\ Hong-Gang Wang $\mathbb{D}^{1,2}$
}

Received: 2 April 2020 / Revised: 4 August 2020 / Accepted: 6 August 2020 / Published online: 17 August 2020

(c) The Author(s), under exclusive licence to ADMC Associazione Differenziamento e Morte Cellulare 2020

\begin{abstract}
Autophagosomal membranes can serve as activation platforms for intracellular death-inducing signaling complexes (iDISCs) to initiate Caspase-8-dependent apoptosis. In this study, we explore the impact of ESCRT-III-dependent phagophore closure on iDISC assemblies and cell death in osteosarcoma and neuroblastoma cells. Inhibition of phagophore closure by conditional depletion of CHMP2A, an ESCRT-III component, stabilizes iDISCs on immature autophagosomal membranes and induces Caspase-8-dependent cell death. Importantly, suppression of the iDISC formation via deletion of ATG7, an E1 enzyme for ubiquitin-like autophagy-related proteins, blocks Caspase-8 activation and cell death following CHMP2A depletion. Although DR5 expression and TRAIL-induced apoptosis are enhanced in CHMP2A-depleted cells, the canonical extrinsic pathway of apoptosis is not responsible for the initiation of cell death by CHMP2A depletion. Furthermore, the loss of CHMP2A impairs neuroblastoma tumor growth associated with decreased autophagy and increased apoptosis in vivo. Together, these findings indicate that inhibition of the ESCRT-III-dependent autophagosome sealing process triggers noncanonical Caspase-8 activation and apoptosis, which may open new avenues for therapeutic targeting of autophagy in cancer.
\end{abstract}

\section{Introduction}

Macroautophagy (hereafter referred to as autophagy) is a highly conserved lysosomal degradation pathway that is responsible for nutrient recycling as well as quality control of proteins and organelles [1]. This catabolic pathway begins with the formation of a small crescent-shaped membrane, known as the phagophore, which elongates and eventually seals to sequester cytoplasmic cargoes within the double-

Edited by: E. Baehrecke

Supplementary information The online version of this article (https:// doi.org/10.1038/s41418-020-00610-0) contains supplementary material, which is available to authorized users.

Yoshinori Takahashi

ytakahashi@pennstatehealth.psu.edu

$\triangle$ Hong-Gang Wang

huw11@psu.edu

1 Department of Pediatrics, Penn State College of Medicine, Hershey, PA 17033, USA

2 Department of Pharmacology, Penn State College of Medicine, Hershey, PA 17033, USA membrane autophagosome. The autophagosome then fuses with the lysosome to degrade the sequestered cargo and recycle the nutrients. Activation of autophagy under the hypoxic and low-nutrient conditions within the tumor microenvironment, as well as in response to cytotoxic drug exposure, supports tumor growth and survival [2]. Therefore, autophagy has emerged as an attractive target for cancer therapy [2-5].

Apoptosis is the most extensively investigated type of regulated cell death, and is characterized by the sequential activation of cysteine-aspartic proteases known as the caspases $[6,7]$. Two major apoptotic pathways, known as the extrinsic and intrinsic pathways, have been shown to activate the "initiator" caspases that cleave and activate the downstream "executioner" caspases, including Caspase-3 and -7. The extrinsic pathway is activated upon the binding of extracellular ligands to cell surface death receptors (DRs), whereas the intrinsic pathway is triggered by mitochondrial outer-membrane permeabilization, which is controlled by the Bcl-2 family of proteins. Once activated, the executioner Caspases cleave hundreds of cellular substrates including poly(ADP-ribose) polymerase (PARP), laminin, and XK-related protein 8 , leading to the acquisition of the apoptotic morphotype [8]. 
Caspase- 8 is an initiator caspase that is required for the extrinsic pathway of apoptosis. Upon the DR-ligand ligation, proCaspase- 8 and the adapter protein FAS-associated protein with death domain (FADD) are recruited to the receptor on the plasma membrane where they form the death-inducing signaling complex (DISC). The DISC formation induces proCaspase- 8 dimerization, self-cleavage, and subsequent activation to initiate the caspase cascade for apoptosis. In addition to this canonical pathway, proCaspase- 8 can also be activated on the autophagosomal membrane through the formation of the intracellular death-inducing signaling complex (iDISC) [9-16]. FADD and the autophagy receptor p62 interact with ATG5 and LC3, respectively, to function as adapters for iDISC assembly to mediate the recruitment and subsequent activation of proCaspase-8. In addition to cleaving executioner caspases, active Caspase- 8 can also directly cleave the pro-apoptotic BH3-only Bcl-2 family protein BID to activate the intrinsic pathway, and cleave the receptorinteracting protein kinase 1 (RIPK1) to suppress necroptosis [7]. However, the progression of autophagy limits Caspase- 8 activation via sequestration of active Caspase- 8 and disassembly of the iDISC arms [10, 17].

The endosomal sorting complex required for transport (ESCRT) machinery that assembles on the inner surface of the membrane neck mediates membrane scission away from the cytoplasm [18]. This so-called "reverse-topology" membrane fission regulates a variety of cellular membrane remodeling processes, including multivesicular body formation, cytokinesis, plasma membrane and lysosomal membrane repair, and nuclear envelope reformation [19]. Recently, we and others identified the ESCRT-III component CHMP2A as a regulator of phagophore closure [20, 21]. Disruption of ESCRT-III-mediated autophagosome sealing leads to the accumulation of immature autophagosomal membranes and prevents the dissociation of ATG proteins [20, 22]. Therefore, we reasoned that inhibition of ESCRT-III-dependent phagophore closure should stabilize iDISC assembly for Caspase-8 activation and redirect pro-tumorigenic autophagy to apoptosis. Indeed, we provide evidence here that CHMP2A depletion induces iDISC-mediated noncanonical Caspase-8 activation on immature autophagosomal membranes and inhibits tumor growth in a mouse xenograft model.

\section{Results}

\section{Inducible depletion of the ESCRT-III component CHMP2A leads to the accumulation of ATG5- and LC3-II-positive phagophores}

To establish a genetic model for studying the effect of inhibiting phagophore closure on cell death, we generated U-2 OS osteosarcoma cells expressing doxycycline (Dox)- inducible CHMP2A shRNA or control non-targeting shRNA. Upon the addition of Dox for $48 \mathrm{~h}$, we observed a reduction in CHMP2A expression to a nearly undetectable level in CHMP2A shRNA-expressing cells (Fig. 1a). Consistent with previous studies [20, 21], CHMP2A depletion inhibited autophagic flux under both basal and starvation conditions, as demonstrated by the impaired lysosomal turnover of the autophagosomal membrane marker LC3-II and an increase in the autophagic substrate p62 (Fig. 1a, b). To verify that inhibition of phagophore closure is responsible for the impaired autophagic flux, we performed the HaloTag-LC3 (HT-LC3) assay, in which autophagosomal membrane-unenclosed and -enclosed HTLC3-II are differentially labeled with membraneimpermeable HaloTag ligands (MIL) and membranepermeable HaloTag ligands (MPL) conjugated with two different fluorescent dyes [20]. As expected, Bafilomycin A1 (BafA1)-induced lysosomal inhibition resulted in an accumulation of MPL-labeled, autophagosome-enclosed LC3-II upon starvation in shNT-expressing control cells, whereas CHMP2A-depleted cells demonstrated an accumulation of MIL-labeled, membrane-unenclosed LC3-II under both basal and starvation conditions (Fig. 1c), indicating a defect in autophagosome completion. The impairment of phagophore closure in Dox-inducible CHMP2A-depleted cells was further confirmed using the phagophore marker ATG5 (Fig. 1d). Collectively, we established an inducible CHMP2A depletion system, where Dox treatment leads to the accumulation of the key iDISC components ATG5, LC3-II, and p62 on autophagosomal membranes.

\section{CHMP2A depletion induces cell death accompanied by cleavage of Caspase-8, Caspase-3, and PARP in osteosarcoma and neuroblastoma cells}

CHMP2A-depleted cells fail to proliferate and eventually undergo cell death [23]. We next determined if the impaired phagophore closure precedes the induction of cell death upon CHMP2A depletion. A time-course analysis showed that the level of LC3-II began to increase at $48 \mathrm{~h}$ after Dox treatment (Fig. 2a), which was accompanied by a gradual increase in cell death as determined by the loss of plasma membrane integrity (YOYO-3-positive) (Fig. 2b) in U-2 OS cells. Consistently, cleavage of the initiator Caspase- 8 and the executor Caspase- 3 was only detected at later time points (60 and $72 \mathrm{~h}$ after Dox treatment) during CHMP2A depletion (Fig. 2a). Moreover, in agreement with our previous observations that nutrient starvation enhances noncanonical Caspase-8 activation on autophagosomal membranes [11], starvation further enhanced the kinetics of CHMP2A depletion-induced cell death (Fig. 2b). To verify that the observed phenotypes are specific to the loss of CHMP2A, the 
a

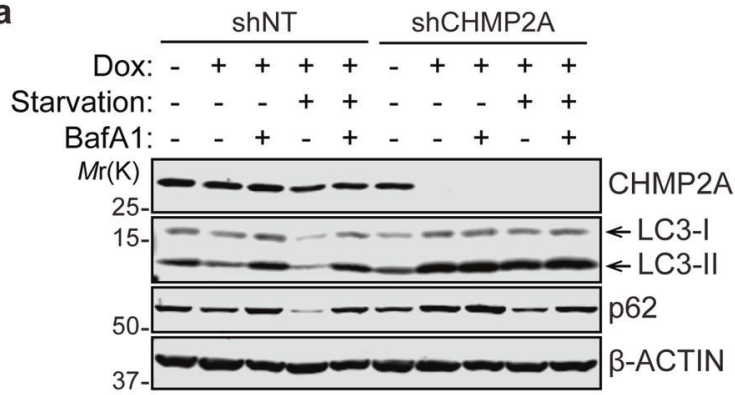

C

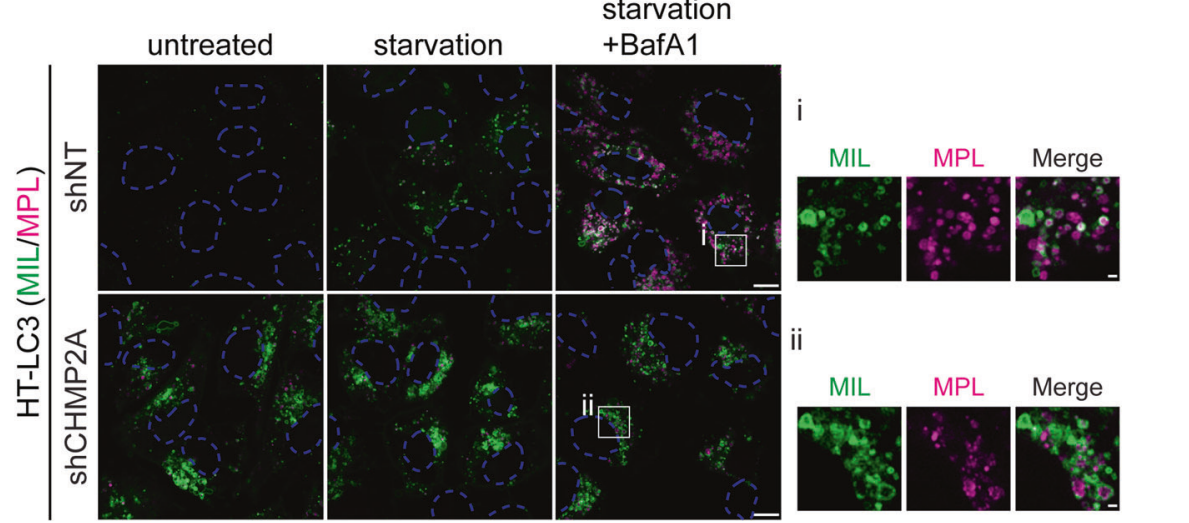

b

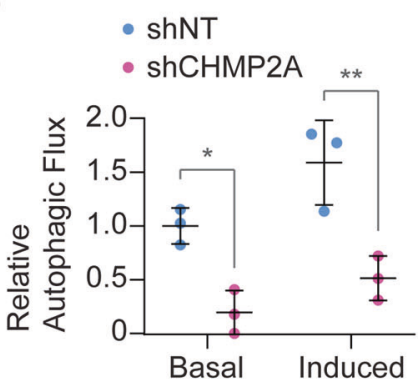

d CFP-ATG5

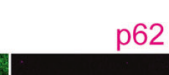

062
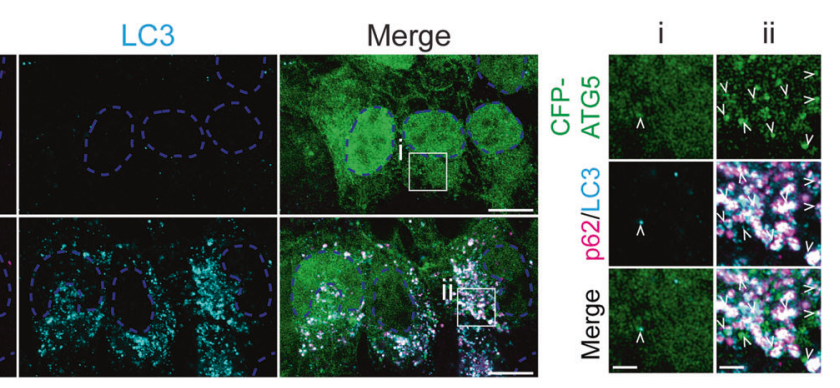

Fig. 1 Inducible depletion of the ESCRT-III component CHMP2A leads to the accumulation of immature autophagosomal membranes. a U-2 OS cells stably expressing inducible non-targeting shRNA (shNT) or CHMP2A shRNA (shCHMP2A) were pretreated with $1 \mu \mathrm{g} / \mathrm{ml}$ doxycycline (Dox) for $48 \mathrm{~h}$, incubated in Dox-containing complete medium or starvation medium in the presence or absence of $100 \mathrm{nM}$ Bafilomycin A1 (BafA1) for $3 \mathrm{~h}$, and subjected to immunoblotting using the indicated antibodies. $\mathbf{b}$ Basal and starvation-induced autophagic flux were calculated as described in "Materials and Methods" and normalized to the value of non-starved shNT-expressing cells $(n=3)$. All values are mean \pm SD. ns not significant; $* p \leq 0.05$;

cells were transduced with shRNA-resistant CHMP2A $\left(\mathrm{CHMP} 2 \mathrm{~A}^{\mathrm{R}}\right.$-myc). We found that the expression of CHMP2A ${ }^{\mathrm{R}}$-myc inhibited shCHMP2A-induced LC3-II and p62 accumulation, Caspase-8/-3 cleavage (Fig. 2c) and activation (Fig. 2d), and cell death under both non-starved and starved conditions (Fig. 2b). Desensitization of CHMP2Adepleted cells to apoptosis by $\mathrm{CHMP} 2 \mathrm{~A}^{\mathrm{R}}$-myc expression was further confirmed by Annexin V/7-amino-actinomycin D (7-AAD) staining followed by flow cytometry (Fig. 2e).

The effect of CHMP2A depletion on Caspase-8/-3 cleavage and cell death was further evaluated using SK-N-AS
${ }^{* *} p \leq 0.01$. The indicated cells were stably transduced with lentiviruses encoding HaloTag-LC3 (HT-LC3) (c) or retroviruses encoding CFPATG5 (d) and treated with $1 \mu \mathrm{g} / \mathrm{ml}$ of doxycycline (Dox) for $54 \mathrm{~h}$. c Cells were starved in the presence or absence of $100 \mathrm{nM}$ BafA1 for 3 $\mathrm{h}$, and subjected to the HT-LC3 assay using Alexa Fluor 660conjugated membrane-impermeable ligand (MIL, green) and tetramethylrhodamine-conjugated membrane-permeable ligand (MPL, magenta) followed by confocal microscopy. d Cells were stained for LC 3 and p62 and analyzed by confocal microscopy. Magnified images in the boxed areas in (c, d) are shown in the right panels (i, ii). The scale bars represent $10 \mu \mathrm{m}$, and $1 \mu \mathrm{m}$ in the magnified images.

neuroblastoma cells and primary human foreskin fibroblasts (HFF-1). Similar to U-2 OS cells, impaired autophagic flux followed by the induction of Caspase-8/-3 cleavage was observed in SK-N-AS cells upon CHMP2A depletion (Fig. 3a). However, despite over 90\% depletion of CHMP2A at the 72-h time-point, the levels of LC3-II and p62 remained unchanged (Fig. 3b) and the induction of cell death was strongly delayed in HFF-1 cells compared to U-2 OS cells (Fig. 3c). As our gene silencing approach did not completely deplete CHMP2A, the residual proteins in the CHMP2A shRNA-expressing cells may be sufficient to induce 
a

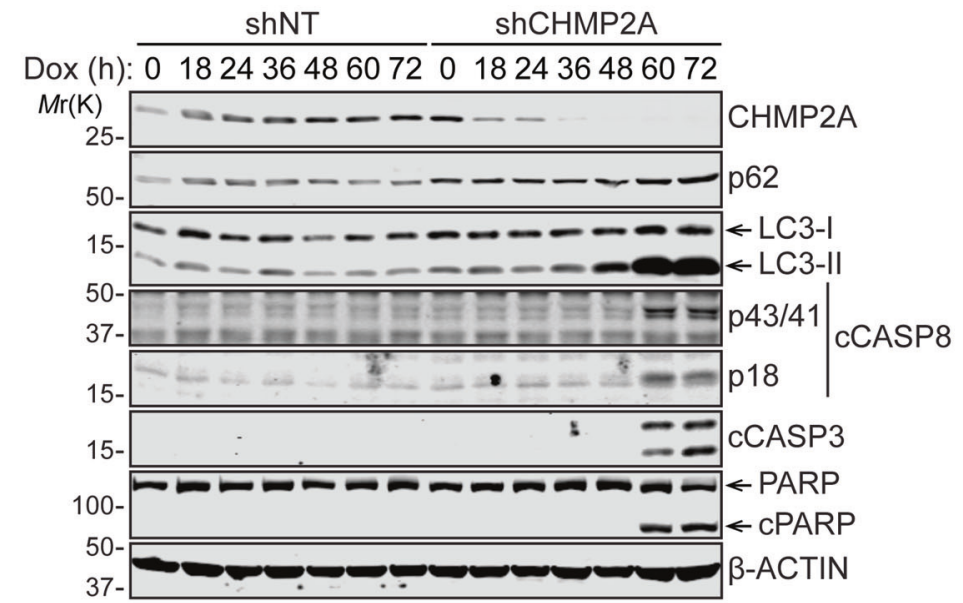

b

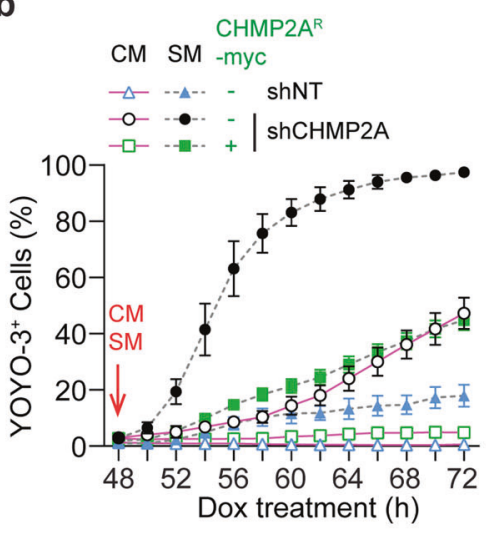

d

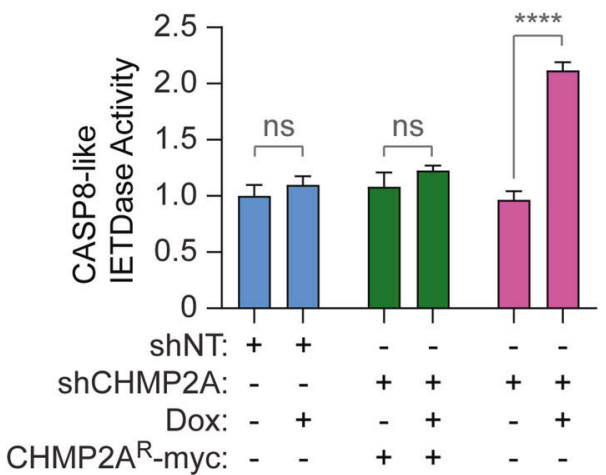

Fig. 2 CHMP2A depletion induces apoptosis in U-2 OS cells. a The indicated U-2 OS cells were treated with $1 \mu \mathrm{g} / \mathrm{ml}$ Dox for the indicated periods of time and analyzed by immunoblotting using the indicated antibodies. cCASP8, cleaved Caspase-8; cCASP3, cleaved Caspase-3; cPARP, cleaved PARP. b Cells were pretreated with $1 \mu \mathrm{g} / \mathrm{ml}$ Dox for $48 \mathrm{~h}$ followed by incubation in Dox-containing complete medium (CM) or starvation medium (SM) in the presence of $0.5 \mathrm{nM}$ YOYO-3 iodide to quantify cells positive for YOYO-3 iodide using the IncuCyte Live-Cell Analysis System. CHMP $2 \mathrm{~A}^{\mathrm{R}}$-myc indicates cells

phagophore closure in HFF-1 cells. To exclude this possibility, we next employed the CRISPR-Cas9 gene editing system to knockout the CHMP2A gene. Five days after CHMP2A gRNA transduction, we observed a strong reduction of CHMP2A in both HFF-1 and U-2 OS cells (Fig. 3d), indicating successful disruption of the CHMP2A gene in the
shNT: $++-\quad++--$

ShCHMP2A: $-\quad++-++$

Dox: -+++++

R-myc: - $-\quad-++++$

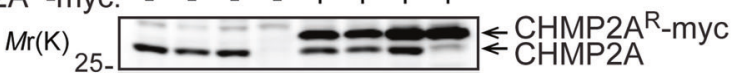

$25-\ldots=-=-\mathrm{CHMP} 2 \mathrm{~A}$

50-

$15-\cdots \div$ - $-2 C 3-1$

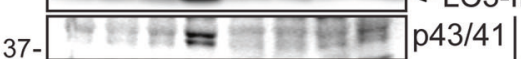

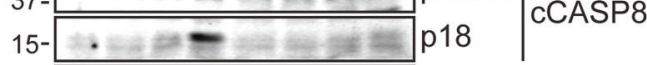

$15-\square \quad$ CCASP3

37- B-ACTIN

e

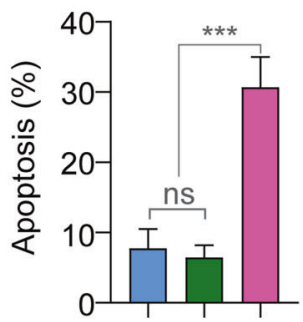

shNT: + - -

ShCHMP2A: -++

CHMP2A $\mathrm{R}^{\mathrm{R} \text { myc: }}$ - +

stably transduced with lentiviruses encoding shRNA-resistant myctagged CHMP2A (CHMP2 $\mathrm{A}^{\mathrm{R}}$-myc). Cells were incubated in the presence or absence of $1 \mu \mathrm{g} / \mathrm{ml}$ Dox for $72 \mathrm{~h}$ and subjected to immunoblotting using the indicated antibodies (c), Caspase- 8 activity assays (d), and Annexin V-APC/7-AAD staining followed by flow cytometry (e). In (d), the Caspase-8-like IETDase activity in each sample was normalized to the value of Dox-untreated shNT-expressing cells. All values are mean $\pm \mathrm{SD}(n=3)$. ns not significant; $* * * p \leq 0.001$; $* * * * p \leq 0.0001$.

majority of the cell population. Consistent with the role of CHMP2A in phagophore closure, knockout of CHMP2A (crCHMP2A) resulted in time-dependent increases in LC3-II and p62 in both HFF-1 and U-2 OS cells. However, unlike U2 OS cells, HFF-1 cells failed to induce a detectable level of Caspase- 8 cleavage and only a mild increase of cleaved 
a

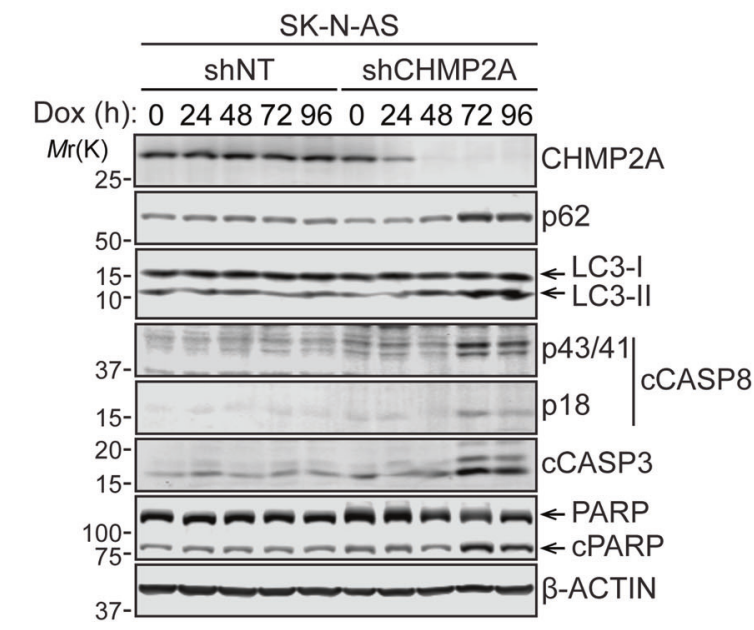

d

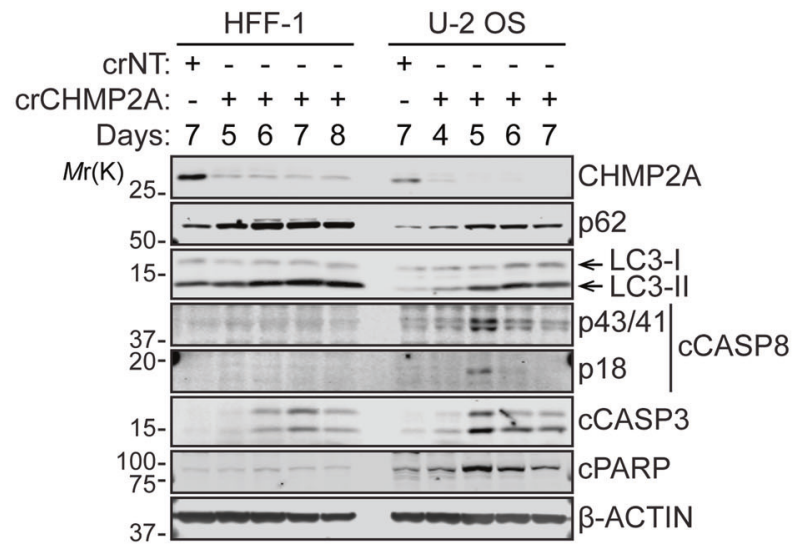

b

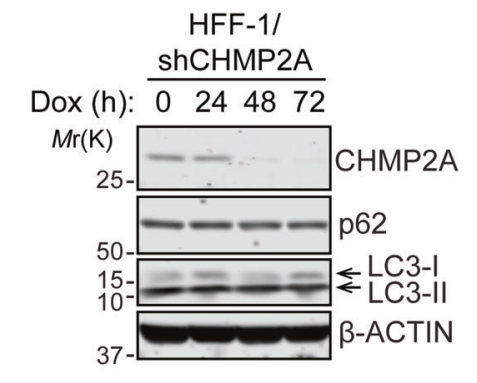

C

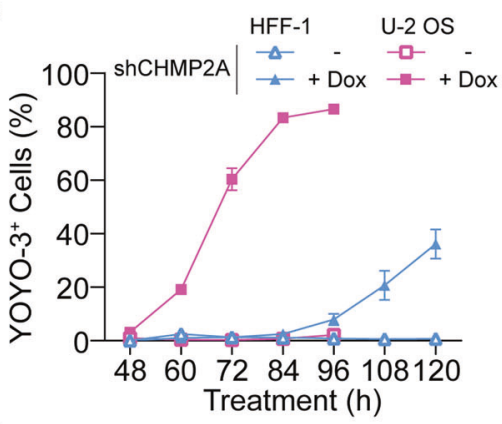

e
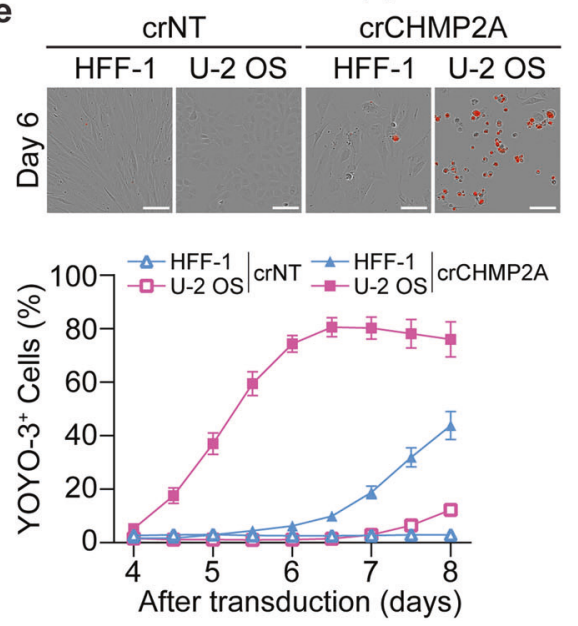

Fig. 3 CHMP2A depletion induces spontaneous cell death in SKN-AS but not HFF-1 cells. a-c SK-N-AS (a) and HFF-1 (b) cells were stably transduced with lentiviruses encoding the indicated inducible shRNAs, treated with $1 \mu \mathrm{g} / \mathrm{ml}$ Dox for the indicated periods of time, and subjected to immunoblotting using the indicated antibodies. $\mathbf{c}$ The indicated inducible shRNA-expressing HFF-1 and U-2 OS cells were pretreated with $1 \mu \mathrm{g} / \mathrm{ml}$ Dox for 72 and $48 \mathrm{~h}$, respectively, incubated in Dox and YOYO-3 iodide-containing complete medium and analyzed using the IncuCyte Live-Cell Analysis System

Caspase-3 was detected, which appeared to be below the threshold for inducing cell death (Fig. 3d, e).

While HFF-1 cells were less sensitive to CHMP2A depletion-induced apoptosis, we found that the basal levels of LC3-II and p62 in HFF-1 cells are much higher than those in U-2 OS cells (Fig. 3d, e). The increase of these proteins is likely due to a slower rate of autophagic flux in HFF-1 cells, as the autophagic inhibitor BafA1 only modestly increased their levels (Fig. S1a). Consistently, immunofluorescence (IF) microscopy showed an accumulation of LC3 and p62-positive $(n=3)$. d, e Three lentiviruses encoding different gRNAs against CHMP2A were pooled and transduced into HFF-1 and U-2 OS cells for $48 \mathrm{~h}$. Cells were then selected with $1 \mu \mathrm{g} / \mathrm{ml}$ puromycin for $38 \mathrm{~h}$ followed by incubation in complete medium for $10 \mathrm{~h}$ (day 4). Cells were cultured for the indicated periods of time and subjected to immunoblotting using the indicated antibodies (d) or incubated in complete medium containing $0.5 \mathrm{nM}$ YOYO-3 iodide and analyzed using the IncuCyte Live-Cell Analysis System with the Cell-by-Cell Analysis module $(n=3)(\mathbf{e})$.

structures whose number appeared to remain unchanged upon short-time exposure to BafA1 in HFF-1 cells (Fig. S1b). To determine the identity of these autophagic structures, we performed the HT-LC3 assay and found an accumulation of $\mathrm{MIL}^{-} \mathrm{MPL}^{+}$mature autophagosomes in HFF-1 cells (Fig. S1c). These results are in agreement with the observation that iDISC activation requires immature autophagosomal membranes (Fig. 4) [10,11] and suggest the importance of a high autophagic flux rate for efficiently inducing apoptosis by CHMP2A depletion. 


\section{Inhibition of iDISC assembly blocks Caspase-8 activation and cell death upon CHMP2A depletion}

To determine if CHMP2A depletion induces Caspase-8 activation on the autophagosomal membrane, we performed the bimolecular fluorescence complementation (BiFC) assay using the catalytically inactive proCaspase- $8(\mathrm{C} 360 \mathrm{~A})$ mutant, as previously described [10]. As expected, we observed an accumulation of BiFC signals that colocalized with LC3 and p62 in CHMP2A-depleted cells (Fig. 4a, b), indicating that proCaspase- 8 dimerization and subsequent activation occur on the phagophore upon CHMP2A depletion. To determine if the autophagosomal membranedependent activation of Caspase- 8 is responsible for the induction of cell death by CHMP2A depletion, we generated ATG7-deficient (crATG7) and Caspase-8-deficient (crCASP8) cells that express inducible shCHMP2A. In agreement with the iDISC model-that proCaspase- 8 is recruited to the immature autophagosomal membrane through the ATG12-ATG5:FADD and LC3-II:p62 arms $[10,11]-$ CHMP2A depletion-induced activation of Caspase- 8 and cleavage of both Caspase- 3 and PARP were inhibited in ATG7-knockout cells, in which the ATG12ATG5 and LC3-phosphatidylethanolamine conjugations required for iDISC formation were disrupted (Fig. 4c, d). Moreover, this ATG conjugation system-dependent activation of Caspase- 8 was responsible for initiation of the cell death cascade, as the loss of Caspase- 8 blocked Caspase- 3 and PARP cleavage despite the accumulation of autophagosomal membrane markers upon CHMP2A depletion (Fig. 4c). The importance of iDISC-mediated Caspase- 8 activation on CHMP2A depletion-induced cell death was further evaluated by Annexin V/7-AAD and YOYO-3 staining under both untreated (Fig. 4e, f) and starvation conditions (Fig. S2a, b). Consistently, the loss of either ATG7 or Caspase- 8 abrogated the induction of apoptosis following CHMP2A depletion. Collectively, these results suggest that CHMP2A depletion induces the noncanonical activation of Caspase- 8 on the phagophore, which triggers activation of the caspase cascade, leading to cell death.

\section{CHMP2A depletion induces cell death independently of the DR5/TRAIL pathway but promotes TRAIL- induced apoptosis}

To determine if CHMP2A depletion also affects the extrinsic pathway of apoptosis, we treated the cells with TRAIL, a well-known inducer of the Caspase-8-mediated extrinsic pathway of apoptosis through its cognate DRs [24]. We found that CHMP2A depletion drastically enhanced cell death, not only in response to nutrient starvation, but also in response to TRAIL treatment (Fig. 5a). Consistently, the TRAIL receptor DR5 was upregulated upon CHMP2A depletion (Fig. 5b). These observations are in agreement with previous studies that demonstrate that autophagymediated ER turnover plays a critical role in overcoming ER stress [25] and that ER stress upregulates both TRAIL and DR5 expression [26, 27]. To determine if the upregulated TRAIL/DR5 pathway contributes to apoptosis following the loss of CHMP2A, cells were treated with the TRAIL neutralizing antibody RIK-2. We observed that TRAILenhanced (Fig. 5c), but not starvation-enhanced (Fig. 5d) or spontaneous (Fig. 5e, f), apoptosis in CHMP2A-depleted cells was inhibited by RIK-2, indicating that the TRAIL/ DR5 pathway is not responsible for the initiation of cell death by CHMP2A depletion. We next examined the involvement of ESCRT-III-dependent plasma membrane repair [28] in CHMP2A depletion-induced cell death. Unlike in necrosis-prone cells, in which CHMP2A silencing promotes RIPK1-dependent necroptotic cell death [28], in U-2 OS cells the RIPK1 inhibitor Nec-1 had little effect on cell death induced by CHMP2A depletion, whereas the pancaspase inhibitor z-VAD-fmk abrogated cell death (Fig. 5g). Moreover, the addition of Nec-1 did not further enhance the $\mathrm{z}$-VAD-fmk-mediated suppression of cell death (Fig. $5 \mathrm{~g}$ ). These results are in agreement with the observation that cell death induced by the loss of CHMP2A was nearly completely inhibited by the loss of Caspase-8 (Fig. 4c-f).

\section{CHMP2A depletion suppresses tumor growth and prolongs the survival of tumor-bearing mice}

To determine if CHMP2A depletion suppresses tumor growth in vivo, a mouse xenograft model was established by subcutaneously xenografting NOD SCID gamma (NSG) mice with either inducible shCHMP2A or shNT cells. We were unable to establish xenograft tumors using U-2 OS cells in NSG mice, so SK-N-AS cells were utilized for all mouse experiments. Once tumors were established, mice began receiving either daily administration of Dox via oral gavage or no treatment. As shown in Fig. 6a, induction of CHMP2A shRNA by Dox treatment suppressed tumor growth compared to both no treatment and shNT tumor groups. Consequently, all of the Dox-treated mice in the shCHMP2A-expressing group survived longer than mice in the untreated or shNT groups, and were all still alive at the study endpoint, at which time all other groups had reached the humane experimental endpoint, which was defined as a tumor volume of $4000 \mathrm{~mm}^{3}$ (Fig. 6b).

We next examined if CHMP2A depletion promotes tumor apoptosis. To minimize sample variability due to individual differences of initial tumor growth, Dox treatment was started when the tumor volume reached $350 \mathrm{~mm}^{3}$. Consistent with the data presented above, shCHMP2Aexpressing tumors failed to grow in the presence of Dox (Figs. 6c and S3). As expected, after 8 days of daily Dox 
a
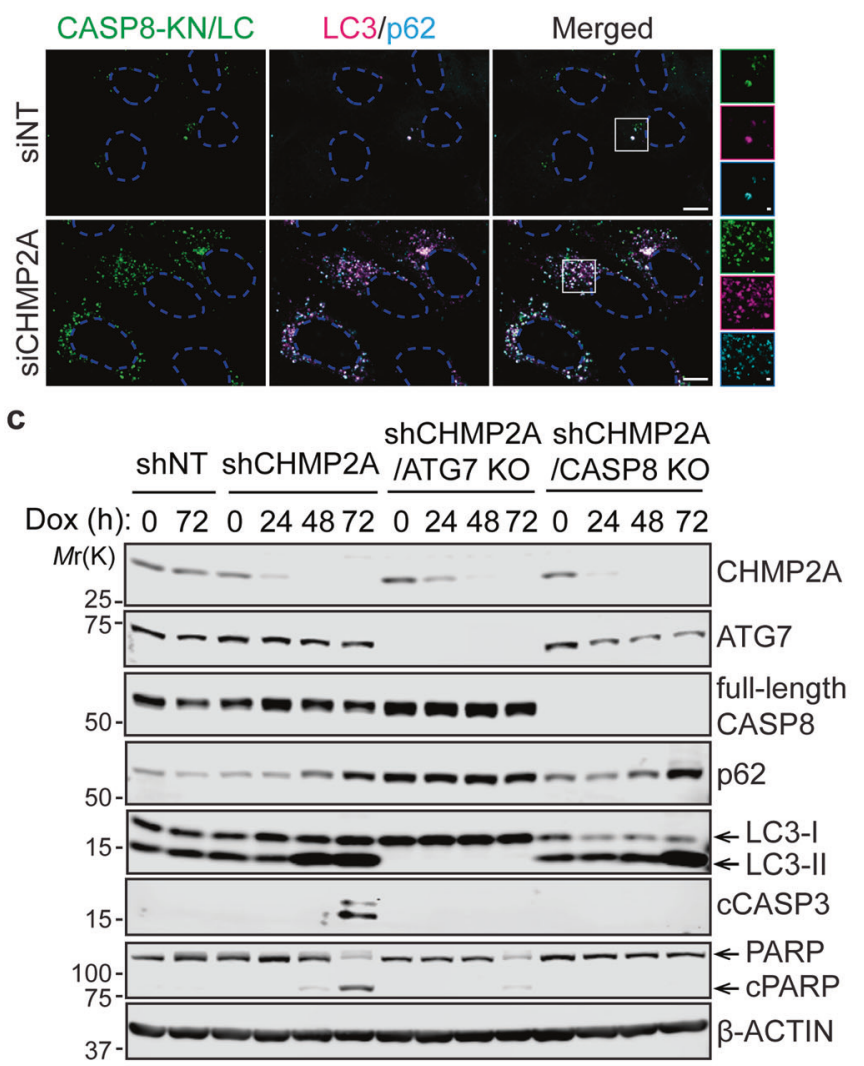

b

\section{(1)}

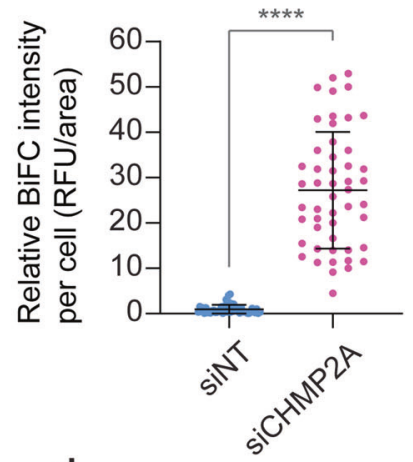

d

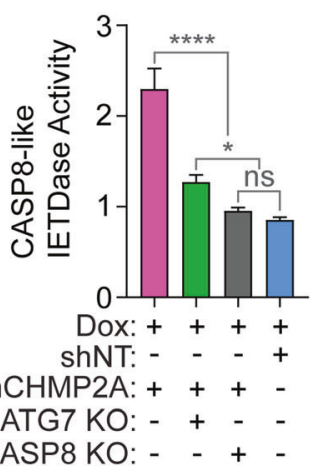

f

e
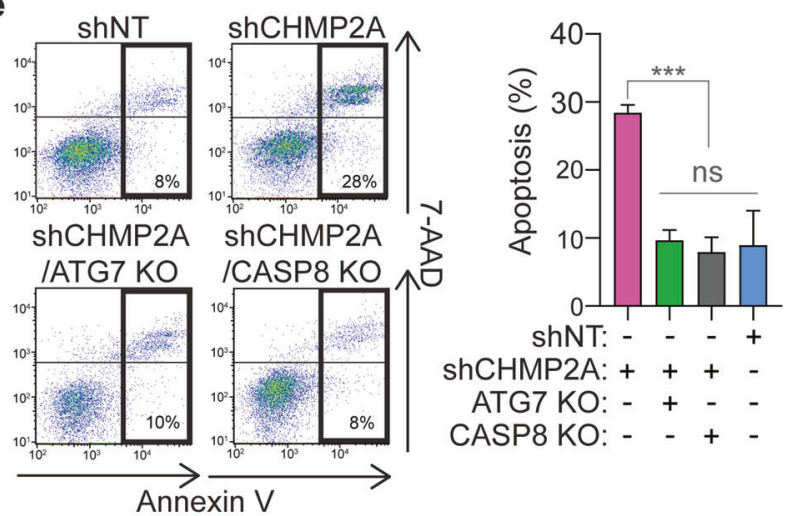

shNT: - - -+

shCHMP2A: +++-

ATG7 KO: - + - -

CASP8 KO: - - + -
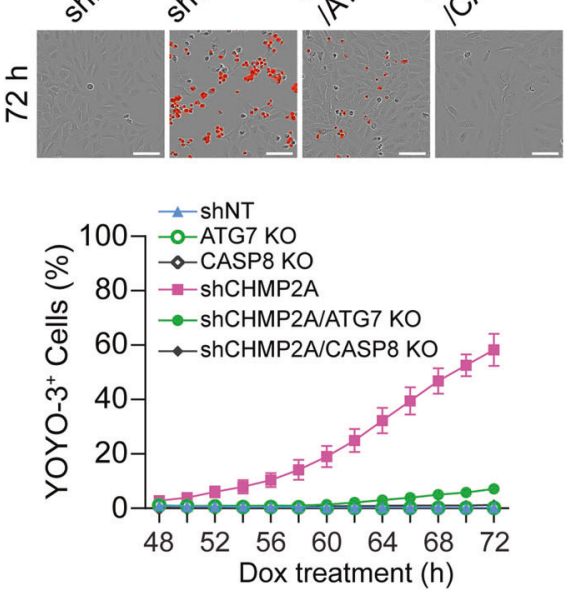

Fig. 4 CHMP2A depletion induces iDISC-mediated apoptosis. a U2 OS cells transduced with lentivirus encoding myc-Caspase-8 (C360A)-KN151 and HA-Caspase-8 (C360A)-LC151 for $72 \mathrm{~h}$ were transfected with the indicated siRNAs for $30 \mathrm{~h}$, stained for LC3 and p62, and analyzed by confocal microscopy. Magnified images in the boxed areas are shown in the right panels. The scale bars represent 10 $\mu \mathrm{m}$, and $1 \mu \mathrm{m}$ in the magnified images. $\mathbf{b}$ The cytoplasmic fluorescence intensities of bimolecular fluorescence complementation (BiFC) complexes in (a) were quantified and shown $(n>50)$. Statistical significance was determined by Mann-Whitney nonparametric $t$-test. The values are mean $\pm \mathrm{SD}$. $* * * * p \leq 0.0001$. c Parental wild-type, ATG7knockout (KO), and CASP8 KO U-2 OS cells stably expressing the

indicated inducible shRNAs were treated with $1 \mu \mathrm{g} / \mathrm{ml}$ Dox for the indicated periods of time, and subjected to immunoblotting using the indicated antibodies. $\mathbf{d}$ Cells were incubated in the presence or absence of $1 \mu \mathrm{g} / \mathrm{ml}$ Dox for $72 \mathrm{~h}$ and the Caspase-8-like IETDase activities were measured and normalized to the value of Dox-untreated shNTexpressing cells $(n=3)$. e Cells treated with $1 \mu \mathrm{g} / \mathrm{ml}$ Dox for $72 \mathrm{~h}$ were subjected to Annexin V-APC/7-AAD staining and analyzed by flow cytometry $(n=3)$. f Cells were pretreated with $1 \mu \mathrm{g} / \mathrm{ml}$ Dox for $48 \mathrm{~h}$, incubated in Dox and YOYO-3 iodide-containing complete medium, and analyzed using the IncuCyte Live-Cell Analysis System $(n=3)$. All values are mean \pm SD. ns not significant; $* p \leq 0.05$; $* * * x \leq 0.001$; $* * * * p \leq 0.0001$. 
a

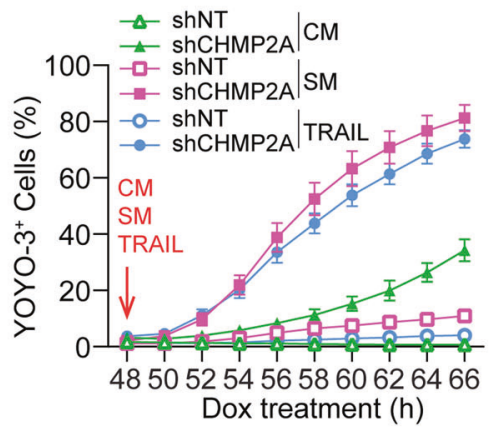

C
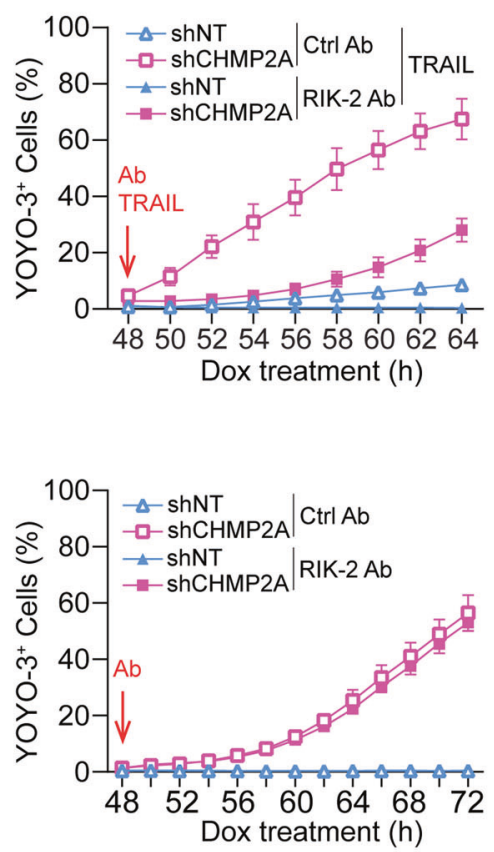

g $24 \mathrm{~h}$ after inhibitor treatment
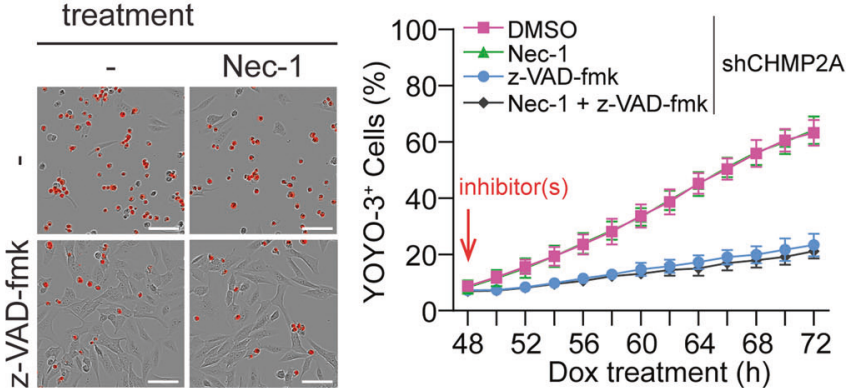

b

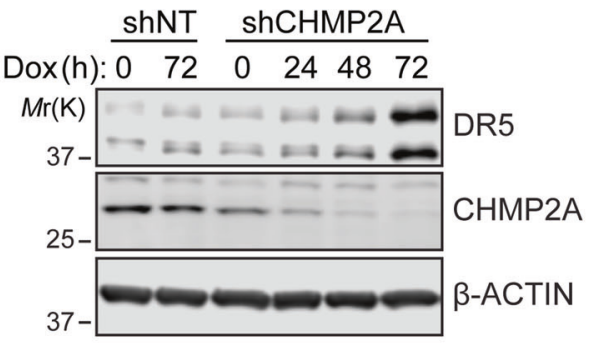

d

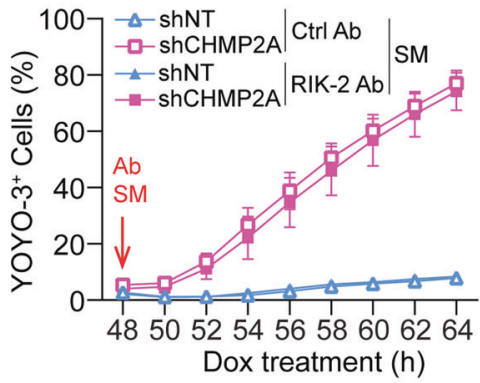

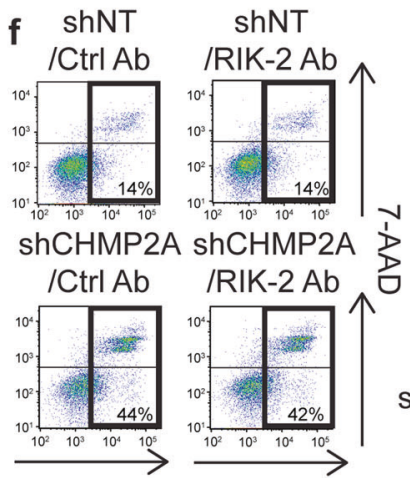

Annexin $\mathrm{V}$

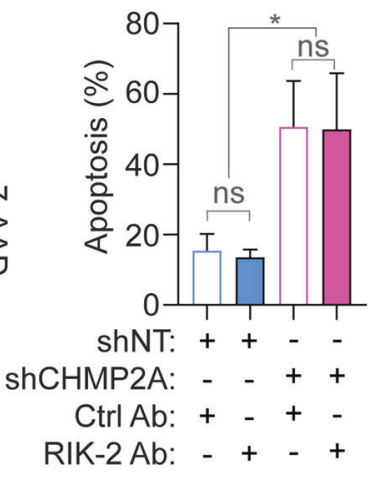

RIK-2 Ab: - + - +
Fig. 5 Upregulation of the death receptor pathway is not responsible for spontaneous and starvation-enhanced apoptosis upon CHMP2A depletion. a The indicated U-2 OS cells were pretreated with $1 \mu \mathrm{g} / \mathrm{ml}$ Dox for $48 \mathrm{~h}$, incubated in Dox and YOYO-3 iodidecontaining starvation medium (SM) or complete medium $(\mathrm{CM})$ in the presence or absence of $100 \mathrm{ng} / \mathrm{ml}$ TRAIL for the indicated periods of time, and analyzed using the IncuCyte Live-Cell Analysis System ( $n$ $=3$ ). b Cells treated with $1 \mu \mathrm{g} / \mathrm{ml}$ Dox for the indicated periods of time were subjected to immunoblotting using antibodies against DR5, CHMP2A, and $\beta$-ACTIN. Cells pretreated with $1 \mu \mathrm{g} / \mathrm{ml}$ Dox for $48 \mathrm{~h}$ were incubated in complete medium containing $100 \mathrm{ng} / \mathrm{ml}$ TRAIL (c), starvation medium (d), or complete medium (e) in the presence of anti- human TRAIL monoclonal antibody RIK-2 or mouse IgG1 isotype control (Ctrl Ab) (100 ng/ml), Dox and YOYO-3 iodide, and analyzed using the IncuCyte Live-Cell Analysis System $(n=3)$. f Cells pretreated with $1 \mu \mathrm{g} / \mathrm{ml}$ Dox for $48 \mathrm{~h}$ were incubated in complete medium containing $100 \mathrm{ng} / \mathrm{ml} \mathrm{RIK}-2$ or $\mathrm{Ctrl} \mathrm{Ab}$ for $24 \mathrm{~h}$ and subjected to Annexin V-APC/7-AAD staining followed by flow cytometry $(n=3)$. g Cells pretreated with $1 \mu \mathrm{g} / \mathrm{ml}$ Dox for $48 \mathrm{~h}$ were incubated in complete medium containing $0.5 \mathrm{nM}$ YOYO- 3 iodide and control DMSO, $20 \mu \mathrm{M}$ of Necrostatin-1 (Nec-1), $20 \mu \mathrm{M}$ z-VAD-fmk, or Nec-1 plus zVAD-fmk for $24 \mathrm{~h}$, and analyzed using the IncuCyte Live-Cell Analysis System $(n=3)$. 
a

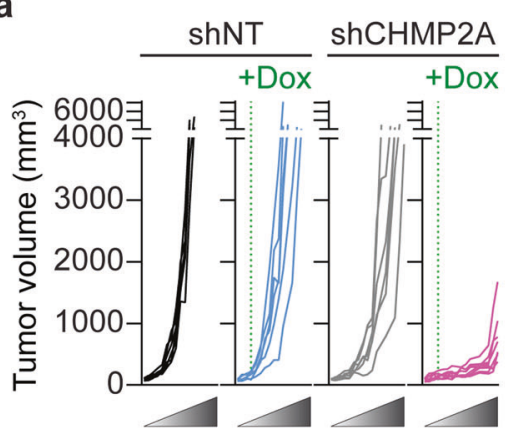

C

d
Days b $-\operatorname{shNT}(n=6) \quad-\operatorname{shCHMP2A}(n=6)$

$-\operatorname{shNT+Dox}(n=6)-\operatorname{shCHMP2A+Dox}(n=8)$

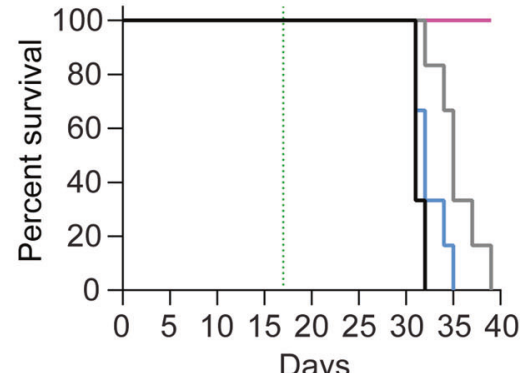

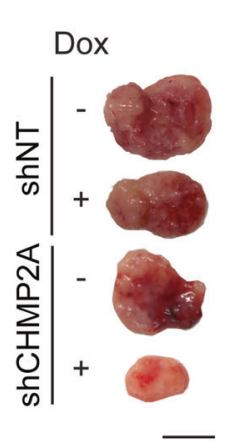

Days

shNT

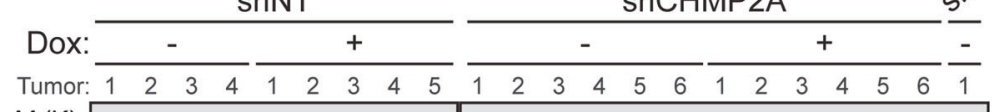

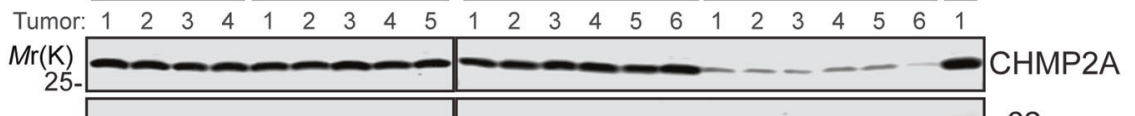

50- $-\ldots-\ldots-\ldots-\ldots$ p

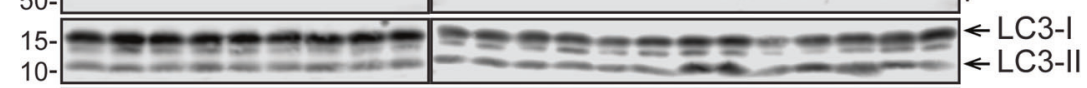

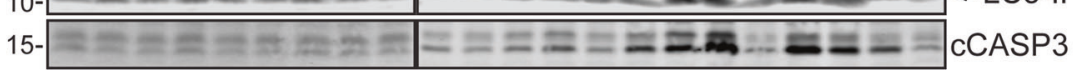

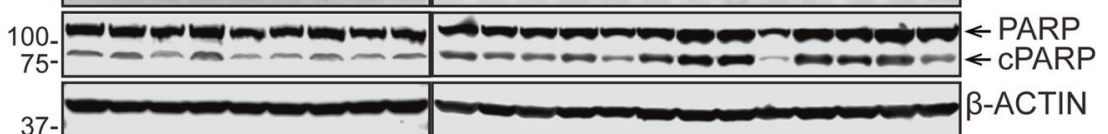

e

$\begin{array}{lllll}\text { CHMP2A p62 LC3-II CCASP3 CPARP } & \end{array}$

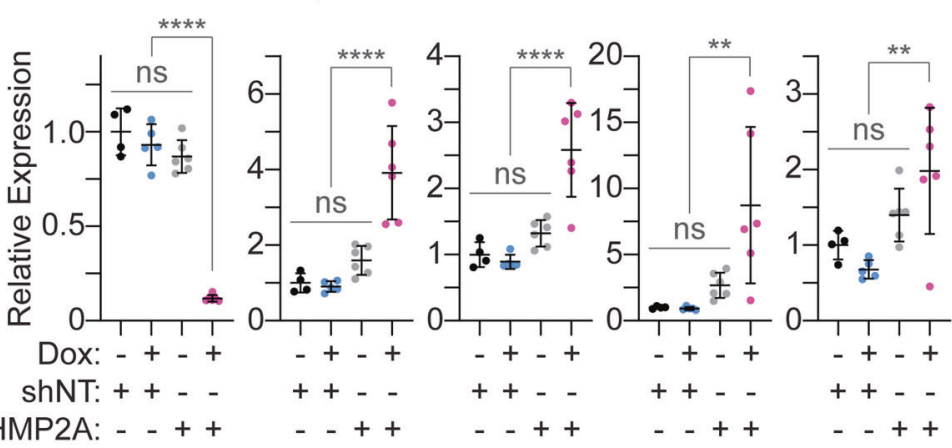

f CCASP3

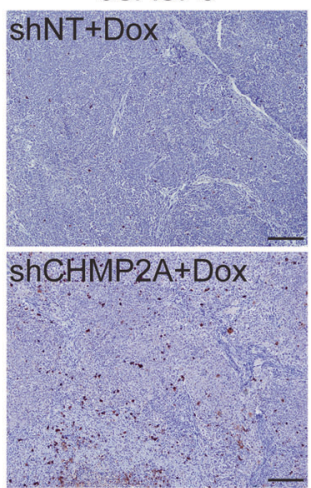

Fig. 6 Inducible depletion of CHMP2A impairs SK-N-AS neuroblastoma tumor growth. Mice bearing the indicated SK-N-AS xenografts received daily administration of Dox beginning 17 days after xenotransplantation (a, b) or when tumor size reached $350 \mathrm{~mm}^{3}$ (c-f). Tumor growth (a) and Kaplan-Meier survival (b) curves of mice in the presence or absence of Dox. The first administration day of Dox (day 17) is indicated by green broken lines. c Representative gross images of tumors dissected from the indicated tumor-bearing mice

treated with or without Dox for 8 days. d Immunoblot analysis of tumor lysates using the indicated antibodies. e CHMP2A, p62, LC3-II, cleaved Caspase-3 (cCASP3), and cleaved PARP (cPARP) levels in (d) were quantified, normalized to $\beta$-ACTIN and shown. All values are mean \pm SD. ns not significant; $* * p \leq 0.01$; $* * * * p \leq 0.0001$. f Representative images of tumor sections stained for cCasp-3. The scale bars in (c, f) represent $1 \mathrm{~cm}$ and $200 \mu \mathrm{m}$, respectively.

administration, we detected a reduction in CHMP2A accompanied by significant increases in LC3-II, p62, cleaved Caspase-3, and cleaved PARP in shCHMP2Aexpressing tumors (Fig. 6d, e). The promotion of Caspase-3 activation by CHMP2A depletion in SK-N-AS tumors was further confirmed by immunohistochemistry (IHC) (Fig. 6f). These observations were not due to the detrimental effects of Dox [29], as the growth rates were similar between the Dox-untreated and -treated inducible shNTexpressing tumors (Figs. 6a and S3).

\section{Discussion}

Noncanonical activation of Caspase- 8 occurs on autophagosomal membranes through its interactions with ATG12- 
ATG5:FADD and p62:LC3 complexes (iDISC) [9-16]. Here, we show that depletion of the autophagosome closure regulator CHMP2A activates the Caspase-8-initiated caspase cascade to induce apoptosis in osteosarcoma and neuroblastoma cells. Disruption of the iDISC assembly by the loss of ATG7 inhibits CHMP2A depletion-induced Caspase-8 activation and subsequent cell death events. The observation that inhibition of phagophore closure by CHMP2A depletion leads to the accumulation of proCaspase- 8 BiFC dimers on autophagosomal membranes is in agreement with previous reports that active Caspase- 8 on the inner leaflet of the autophagosomal membrane is subjected to lysosomal degradation [30], whereas ATG12-ATG5 and LC3-II on the outer leaflet dissociate from the membrane upon autophagosome completion [31]. Notably, the level of cell death following nutrient starvation is much greater in CHMP2Adepleted cells compared to ATG7-depleted or CHMP2A/ ATG7 double-depleted cells. These results support the idea that inhibition of phagophore closure not only suppresses the pro-survival function of autophagy but also stabilizes iDISCs to induce cell death [10]. Therefore, targeting autophagy at the autophagosome sealing step may represent an attractive therapeutic option to accelerate cancer cell death by simultaneously blocking cytoprotective autophagy and activating iDISC-mediated apoptosis.

Unlike U-2 OS and SK-N-AS cancer cells, the effects of CHMP2A depletion on Caspase-8 activation and cell death induction are found to be nearly negligible in HFF-1 human fibroblasts, at least in the short term. Although the precise underlying mechanism remains to be characterized, we detect higher basal levels of p62 and LC3-II in the fibroblasts, which do not further accumulate upon shortterm knockdown of CHMP2A or exposure to BafA1. As iDISC formation requires the phagophore or nascent autophagosomal membrane, a lower rate of autophagosome biogenesis would be a limiting factor for iDISC-mediated apoptosis.

ESCRT-III dysfunction mediated by depletion of CHMP4B or by overexpression of the frontotemporal dementia linked to the chromosome 3-associated CHMP2B mutant has been reported to induce neuronal cell loss accompanied by excess accumulation of LC3-positive autophagosomal membranes [32]. Of note, neuronal cell loss induced by the ESCRT-III inhibition is delayed by the knockdown of ATG5/7 [33]. As neither impaired phagophore closure nor cell death is induced by the loss of CHMP2B [20, 34], the disease-associated CHMP2B mutant may act in a dominant-negative fashion to inhibit the CHMP2A-dependent autophagosome sealing process. Moreover, given the involvement of CHMP4B in phagophore closure [21], stabilization of the iDISC arms could be a mechanism underlying the induction of neuronal cell death in frontotemporal dementia.
We found that the loss of CHMP2A also upregulates DR5 expression and enhances TRAIL sensitivity. The observation that RIK-2 desensitizes CHMP2A-depleted cells to TRAIL-induced cell death, but not to starvationinduced or spontaneous cell death, indicates that CHMP2A inhibition can initiate the iDISC-mediated noncanonical cell death cascade independently of, while amplifying, the canonical extrinsic and intrinsic pathways of apoptosis. In flies, mutations in the gene encoding the ESCRT-II component vps 25 induce autophagosomal membrane accumulation [35] and cell death [36-38], similar to CHMP2A depletion in human cells. Interestingly, the fly vps 25 mutant upregulates JNK signaling [36], which has been reported to promote DR5 expression [39] and amplify the Caspase-8initiated cell death cascade [40]. Given the role of autophagy in TRAIL resistance [41] and the limited efficacy of TRAIL therapy [42], our future goal is to determine if iDISC activation mediated by inhibition of ESCRT-IIIdependent phagophore closure synergizes with death ligands to effectively induce cancer cell death in vivo, which may ultimately facilitate the development of autophagy-targeted therapeutics for cancer treatment.

\section{Materials and methods}

\section{Reagents}

The following antibodies were used for IF, immunoblotting (IB), and IHC: mouse antibodies against $\beta$-ACTIN (IB, SigmaAldrich, A5441, 1:10,000), MAP1LC3B (IF, MBL International, M152-3, 1:200 (Fig. 1d)); rabbit antibodies against ATG7 (IB, Cell Signaling, 8558, 1:1,000), CHMP2A (IB, Proteintech, 10477-1-AP, 1:1,000), cleaved Caspase-3 (IB/ IHC, Cell Signaling, 9661, 1:1,000), cleaved Caspase-8 (IB, Cell Signaling, 9496, 1:1,000), MAP1LC3B (IB, Novus, NB100-2220, 1:3,000; IF, Cell Signaling, 3868, 1:200 (Figs. 4a and S1b)), PARP (IB, Cell Signaling, 9542, 1:1,000), ProCaspase-8 (IB, Abcam, ab108333, 1:1,000); guinea pig antibody against p62 (IB, American Research Products, 03GP62-C, 1:4,000). SMARTvector Inducible Lentiviral Nontargeting (VSC11654) and Human CHMP2A (V3SH11252226090300) shRNAs, and ON-TARGETplus SMART Pool Non-targeting (D-001810-10) and Human CHMP2A (L020247-01) siRNAs were purchased from Horizon Discovery. pCDH1-CMV-HaloTag-LC3-SV40-Hygro and sgCHMP2AlentiCRISPRv2 plasmids were generated as previously described [23]. sgAtg7-Cas9-2A-GFP (sc-400997) and sgCaspase-8Cas9-2A-GFP (sc-400147) plasmids were purchased from Santa Cruz Biotechnology. The lentiviral vectors encoding CHMP2A shRNA-resistant CHMP2A-myc (pCDH1-CMVCHMP2A ${ }^{\mathrm{R}}$-myc-SV40-hygro), myc-Caspase-8 (C360A)KN151 (the N-terminal fragment (1-151) of mKate) (pCDH1- 
myc-Caspase-8(C360A)-KN151-SV40-hygro), and HACaspase-8 (C360A)-LC151 (the C-terminal fragment (152-232) of mLumin) (pCDH1-HA-Caspase-8(C360A)LC151-SV40-hygro) were generated by Gibson Assembly. The following linker sequences are introduced between: CHMP2A and myc, $\mathrm{N}^{\prime}$-(CHMP2A)-GGGGS-(myc)-C'; myc, Caspase-8 (C360A), and KN151, N'-(myc)-LM AMEARIRST-(CASP8)-GTGGGGSGGGGS-(KN151)-C';

HA, Caspase-8 (C360A), and LC151, N'-(HA)-LMAMEARIRST-(CASP8)-GTGGGGSGGGGS-(LC151)-C'. The following silence mutations were introduced to generate $\mathrm{shRN}$ A-resistant CHMP2A: wild type: 5'-TGCGCAAGTTT GTATTGAT-3', nucleotide 217-236; mutant: 5'-TGCGCAA GTTTGTATTGAT-3' (mutated nucleotides are underlined). pCMRX-IP/SECFP-mATG5 [43] was a gift from Noboru Mizushima (Addgene \#58744). All other reagents were obtained from the following sources: 7-AAD (BioLegend, 420404); APC Annexin V (BioLegend, 640941); BafA1 (LC Laboratories, B-1080); bovine serum albumin (EMD Millipore, 126575); carbobenzoxy-valyl-alanyl-aspartyl-[Omethyl]-fluoromethylketone (z-VAD-fmk) (MedChemExpress, HY-16658); Caspase-Glo 8 Assay kit (Promega, G8200); Dimethyl sulfoxide (DMSO) (Sigma-Aldrich, D2438); Dox hyclate (SigmaAldrich, D9891); Human TRAIL (Shenandoah Biotechnology, 100-115); Lipofectamine RNAiMAX (Thermo Fisher Scientific, 13778075); Matrigel Membrane Matrix (Thermo Fisher Scientific, CB-40234); MIL (Promega, Alexa Fluor 660-conjugated, G8471); MPL (Promega, tetramethylrhodamine-conjugated, G8251); normal goat serum (NGS) (Sigma-Aldrich, G9023); Necrostatin-1 (Sigma-Aldrich, N9037); Nucleofector Kit V (Lonza, VCA-1003); paraformaldehyde (PFA) (Electron Microscopy Sciences, 15710); Purified anti-human CD253 (TRAIL) [RIK-2] (BioLegend, 308208); Purified Mouse IgG1, $\kappa$ Isotype Ctrl Antibody (BioLegend, 400123); XF Plasma Membrane Permeabilizer (XF-PMP) (Seahorse Bioscience, 102504-100); YOYO-3 Iodide (Thermo Fisher Scientific, Y3606).

\section{Cell culture, transfection, and viral transduction}

U-2 OS cells were maintained in McCoy's 5A Medium supplemented with $10 \%$ fetal bovine serum (FBS) and $1 \mathrm{x}$ Antibiotic Antimycotic Solution (AA) (Corning, 30-004CI). SK-N-AS and 293T/17 cells were maintained in Dulbecco's Modification of Eagle's Medium (DMEM) supplemented with $10 \%$ FBS and 1x AA. HFF-1 cells were maintained in DMEM supplemented with $15 \%$ FBS and 1x AA. All cells were obtained from American Type Culture Collection. To induce autophagy, cells were rinsed with Dulbecco's phosphate-buffered saline (PBS) and incubated with amino acid-free DMEM (Wako, 048-33575). For siRNA-mediated gene silencing, the indicated siRNAs were transfected into cells using Lipofectamine RNAiMAX transfection reagent according to the manufacturer's protocol. Recombinant lentiviruses were produced in 293T/17 cells and transduced into U-2 OS, SK-N-AS, and HFF-1 cells as described previously [23]. ATG7 and CASP8 knockout U-2 OS cells were generated by CRISPR/Cas9mediated genome editing as described previously [44]. Briefly, cells were nucleofected with sgAtg7-Cas9-2A-GFP or sgCaspase-8-Cas9-2A-GFP for $48 \mathrm{~h}$ followed by flow cytometry sorting for GFP-positive cells, cultured for 2 weeks in complete medium, and resorted for GFPnegative cells. Single clones were then isolated by limited dilution and gene silencing was confirmed by IB. Five (ATG7 KO) or four (CASP8 KO) clones were pooled together and used for the experiments.

\section{Immunoblotting}

Total cell lysates and tissue homogenates were prepared in radio-immunoprecipitation assay buffer $(150 \mathrm{mM} \mathrm{NaCl}, 10$ $\mathrm{mM}$ Tris- $\mathrm{HCl}, \mathrm{pH} 7.4,0.1 \%$ SDS, $1 \%$ Triton $\mathrm{X}-100,1 \%$ Deoxycholate, $5 \mathrm{mM}$ EDTA, $\mathrm{pH}$ 8.0) containing protease (Sigma-Aldrich, P8340) and phosphatase (Sigma-Aldrich, P5726) inhibitors, and subjected to SDS-PAGE followed by IB with the indicated antibodies. The signal intensities were quantified using the Image Studio version 5 software (LICOR Biotechnology).

\section{Autophagy assays}

HT-LC3 autophagosome completion assays and IB-based detection of autophagic flux were performed as described previously [23]. Briefly, for the HT-LC3 assay, cells stably expressing HT-LC3 were incubated in 1× MAS buffer (220 $\mathrm{mM}$ mannitol, $70 \mathrm{mM}$ sucrose, $10 \mathrm{mM}$ KH2PO4, $5 \mathrm{mM}$ $\mathrm{MgCl} 2,2 \mathrm{mM}$ HEPES, and $1 \mathrm{mM}$ EGTA) containing $3 \mathrm{nM}$ XF-PMP and $3.5 \mu \mathrm{M}$ MIL-AF660 at $37^{\circ} \mathrm{C}$ for $15 \mathrm{~min}$, fixed in $4 \%$ PFA for $5 \mathrm{~min}$, washed three times in PBS, and incubated with $5 \mu \mathrm{M}$ TMR for $30 \mathrm{~min}$ at room temperature. After washing five times in PBS, cells were analyzed by confocal microscopy. For the autophagic flux assay, signal intensities were quantified using Image Studio version 5 software (LI-COR Biotechnology) and the level of LC3-II in each lane was normalized to the respective value of $\beta$ actin. Autophagic flux was calculated by subtracting normalized LC3-II values in the group without BafA1 from those in the group with BafA1 in complete and starvation medium for basal and induced autophagic flux, respectively.

\section{Confocal microscopy}

Cells were plated on Lab-Tek II Chambered Coverglass, Chamber Slide (Nunc, 154941). Immunofluorescence was performed as follows: for the detection of LC3, p62, and 
CFP-ATG5, cells were fixed in 4\% PFA-PBS for $10 \mathrm{~min}$ and permeabilized in $100 \mu \mathrm{g} / \mathrm{ml}$ digitonin for $5 \mathrm{~min}$. For the BiFC assay, U-2 OS cells were transduced with lentivirus encoding myc-Caspase-8 (C360A)-KN151 and HACaspase-8 (C360A)-LC151 for $72 \mathrm{~h}$, transfected with the indicated siRNAs for $30 \mathrm{~h}$, fixed and permeabilized in methanol at $-20{ }^{\circ} \mathrm{C}$ for $10 \mathrm{~min}$. Cells were then blocked in $10 \%$ NGS for $1 \mathrm{~h}$, and incubated with the indicated primary antibodies overnight at $4{ }^{\circ} \mathrm{C}$ followed by incubation with the secondary antibodies for $1 \mathrm{~h}$. Leica AOBS SP8 laser scanning confocal microscope (oil-immersion [1.2 numerical aperture] lens) with highly sensitive HyD detectors and Leica image acquisition software LAX was used to obtain fluorescence images. Acquired images were deconvolved using Huygens deconvolution software (Scientific Volume Imaging), and analyzed using Imaris software (Bitplane) and Volocity software (PerkinElmer) without gamma adjustment. PBS was used as imaging medium.

\section{Cell death assays}

For the YOYO-3 iodide staining-based live/dead assay, cells were plated in a 96-well plate and incubated in induction medium (complete medium in the presence or absence of 1 $\mu \mathrm{g} / \mathrm{ml}$ Dox) for $48 \mathrm{~h}$. The medium was then replaced with fresh induction medium containing $0.5 \mu \mathrm{M}$ YOYO-3 iodide. Fluorescence and phase images were obtained at 2- or 12-h intervals using the IncuCyte S3 Live-Cell Analysis System (Essen BioScience; 10× objective) and analyzed using the Basic Analyzer or Cell-by-Cell Analysis (Essen BioScience, 9600-0031) modules of the IncuCyte S3 Software (Essen BioScience, Version 2019A). For the Caspase-8 activity assay, cells were plated on a 384-well plate and incubated in induction medium for $72 \mathrm{~h}$. Caspase-8-like IETDase activity was measured using the Caspase-Glo 8 Assay kit according to the manufacturer's protocol. For flow cytometry-based cell death analysis, cells were washed once with ice-cold staining buffer (10 mM HEPES, pH 7.4, $140 \mathrm{mM} \mathrm{NaCl}, 2.5$ $\mathrm{mM} \mathrm{CaCl}$ ), incubated in staining buffer containing APC Annexin V (1:40 dilution) and 7-AAD (1:40 dilution) for 10 min on ice, and analyzed using a BD FACSCanto flow cytometer (BD Biosciences, 657338) and FlowJo Version 10.5.3 software (FlowJo).

\section{Tumor xenograft}

To establish a tumor xenograft model, SK-N-AS cells were harvested, rinsed once with $\mathrm{PBS}$, resuspended in $50 \%$ Matrigel Membrane Matrix at a concentration of $2 \times 10^{7}$ cells $/ \mathrm{ml}$, and subcutaneously injected $\left(4 \times 10^{6}\right.$ cells in 200 $\mu \mathrm{l} /$ flank) into the flank of 7-9-week-old NSG male and female mice (Jackson Laboratory, 005557). Seventeen days after xenotransplantation (Fig. 6a, b) or when tumor volume reached $350 \mathrm{~mm}^{3}$ (Figs. 6c-f and $\mathrm{S} 3$ ), mice in the treatment groups began a daily regimen of $25 \mathrm{mg} / \mathrm{kg}$ Dox diluted in water by oral gavage, which continued for the duration of the experiment. Mouse body weight and tumor volume were measured every 2 days. Tumor volume was calculated as $\left(\pi L W^{2} / 6\right)$ using length $(L)$ and width $(W)$ caliper measurements. The humane endpoint was defined as a tumor volume of $4000 \mathrm{~mm}^{3}$. All animal studies were performed in accordance with the guidelines of the Institutional Animal Care and Use Committee at the Penn State College of Medicine.

\section{Immunohistochemistry}

Tumor tissues were fixed in $10 \%$ formalin, embedded in paraffin, and sectioned at $6 \mu \mathrm{m}$ thickness. Sections were mounted on charged slides, deparaffinized, boiled in citrate buffer $(10 \mathrm{mM}$ sodium citrate, $0.05 \%$ tween $20, \mathrm{pH}$ 6.0) for antigen retrieval, incubated with $3 \%$ hydrogen peroxide for endogenous peroxide inactivation, blocked in $10 \%$ NGS for $1 \mathrm{~h}$, incubated with an anti-cleaved Caspsase- 3 overnight at $4{ }^{\circ} \mathrm{C}$, stained using the VECTASTAIN $\mathrm{ABC}$ kit (Vector Laboratories, PK-6101) and the ImmPACT DAB Peroxidase Substrate (Vector Laboratories, SK-4105), counterstained with hematoxylin (Sigma-Aldrich, 1051740500), and analyzed using an Olympus BX53 microscope.

\section{Statistical analyses}

Statistical significance was determined using Graph Pad Prism 7.0. Threshold for statistical significance for each test was set at $95 \%$ confidence $(p<0.05)$. All data from in vitro studies are representative of three independent experiments. For animal studies, the sample size was determined using a two-sample $t$-test analysis at the test power of 0.80 , set significance level $\alpha=0.05$, and an effect size based on preliminary experiments. The age- and sex-matched mice were randomly assigned to control and treatment groups. No data were excluded from analysis.

Acknowledgements This work was supported by the NIH grants GM127954 and CA222349, the Lois High Berstler Research Endowment Fund, and the Four Diamonds Fund. Confocal images were generated using the Leica SP8 microscope (NIH Shared Instrumentation grant S10OD010756-01A1) located in the Penn State College of Medicine Microscopy Imaging Core Facility.

\section{Compliance with ethical standards}

Conflict of interest The authors declare that they have no conflict of interest.

Publisher's note Springer Nature remains neutral with regard to jurisdictional claims in published maps and institutional affiliations. 


\section{References}

1. Mizushima N, Levine B, Cuervo AM, Klionsky DJ. Autophagy fights disease through cellular self-digestion. Nature. 2008;451:1069-75.

2. Dower CM, Wills CA, Frisch SM, Wang HG. Mechanisms and context underlying the role of autophagy in cancer metastasis. Autophagy. 2018;14:1110-28.

3. Kimmelman AC, White E. Autophagy and tumor metabolism. Cell Metab. 2017;25:1037-43.

4. Towers CG, Wodetzki D, Thorburn A. Autophagy and cancer: modulation of cell death pathways and cancer cell adaptations. J Cell Biol. 2020;219:e201909033.

5. Amaravadi RK, Kimmelman AC, Debnath J. Targeting autophagy in cancer: recent advances and future directions. Cancer Discov. 2019;9:1167-81.

6. Tang D, Kang R, Berghe TV, Vandenabeele P, Kroemer G. The molecular machinery of regulated cell death. Cell Res. 2019;29:347-64.

7. Green DR. The coming decade of cell death research: five riddles. Cell. 2019;177:1094-107.

8. McArthur K, Kile BT. Apoptotic caspases: multiple or mistaken identities? Trends Cell Biol. 2018;28:475-93.

9. Deegan S, Saveljeva S, Logue SE, Pakos-Zebrucka K, Gupta S, Vandenabeele P, et al. Deficiency in the mitochondrial apoptotic pathway reveals the toxic potential of autophagy under ER stress conditions. Autophagy. 2014;10:1921-36.

10. Young MM, Takahashi Y, Khan O, Park S, Hori T, Yun J, et al. Autophagosomal membrane serves as platform for intracellular death-inducing signaling complex (iDISC)-mediated caspase-8 activation and apoptosis. J Biol Chem. 2012;287: $12455-68$.

11. Tang Z, Takahashi Y, Chen C, Liu Y, He H, Tsotakos N, et al. Atg2A/B deficiency switches cytoprotective autophagy to noncanonical caspase-8 activation and apoptosis. Cell Death Differ. 2017;24:2127-38.

12. Huang S, Okamoto K, Yu C, Sinicrope FA. p62/sequestosome-1 up-regulation promotes ABT-263-induced caspase-8 aggregation/ activation on the autophagosome. J Biol Chem. 2013;288:33654-66.

13. Jiang $\mathrm{H}$, White EJ, Rios-Vicil $\mathrm{CI}, \mathrm{Xu}$ J, Gomez-Manzano $\mathrm{C}$, Fueyo J. Human adenovirus type 5 induces cell lysis through autophagy and autophagy-triggered caspase activity. J Virol. 2011;85:4720-9.

14. Pan JA, Ullman E, Dou Z, Zong WX. Inhibition of protein degradation induces apoptosis through a microtubule-associated protein 1 light chain 3-mediated activation of caspase-8 at intracellular membranes. Mol Cell Biol. 2011;31:3158-70.

15. Bell BD, Leverrier S, Weist BM, Newton RH, Arechiga AF, Luhrs KA, et al. FADD and caspase- 8 control the outcome of autophagic signaling in proliferating T cells. Proc Natl Acad Sci USA. 2008;105:16677-82.

16. Laussmann MA, Passante E, Dussmann H, Rauen JA, Wurstle ML, Delgado ME, et al. Proteasome inhibition can induce an autophagy-dependent apical activation of caspase-8. Cell Death Differ. 2011;18:1584-97.

17. Zhang YB, Zhao W, Zeng RX. Autophagic degradation of caspase-8 protects U87MG cells against $\mathrm{H} 2 \mathrm{O} 2$-induced oxidative stress. Asian Pac J Cancer Prev. 2013;14:4095-9.

18. Schoneberg J, Lee IH, Iwasa JH, Hurley JH. Reverse-topology membrane scission by the ESCRT proteins. Nat Rev Mol Cell Biol. 2017;18:5-17.

19. Vietri M, Radulovic M, Stenmark H. The many functions of ESCRTs. Nat Rev Mol Cell Biol. 2020;21:25-42.
20. Takahashi Y, He H, Tang Z, Hattori T, Liu Y, Young MM, et al. An autophagy assay reveals the ESCRT-III component CHMP2A as a regulator of phagophore closure. Nat Commun. 2018;9:2855.

21. Zhen Y, Spangenberg H, Munson MJ, Brech A, Schink KO, Tan $\mathrm{KW}$, et al. ESCRT-mediated phagophore sealing during mitophagy. Autophagy. 2020;16:826-41.

22. Zhou F, Wu Z, Zhao M, Murtazina R, Cai J, Zhang A, et al. Rab5dependent autophagosome closure by ESCRT. J Cell Biol. 2019;218:1908-27.

23. Takahashi Y, Liang X, Hattori T, Tang Z, He H, Chen H, et al. VPS37A directs ESCRT recruitment for phagophore closure. J Cell Biol. 2019;218:3336-54.

24. Tummers B, Green DR. Caspase-8: regulating life and death Immunol Rev. 2017;277:76-89.

25. Abdrakhmanov A, Gogvadze V, Zhivotovsky B. To eat or to die: deciphering selective forms of autophagy. Trends Biochem Sci. 2020;45:347-64.

26. He Q, Lee DI, Rong R, Yu M, Luo X, Klein M, et al. Endoplasmic reticulum calcium pool depletion-induced apoptosis is coupled with activation of the death receptor 5 pathway. Oncogene. 2002;21:2623-33.

27. Yamaguchi $\mathrm{H}$, Wang HG. CHOP is involved in endoplasmic reticulum stress-induced apoptosis by enhancing DR5 expression in human carcinoma cells. J Biol Chem. 2004;279:45495-502.

28. Gong YN, Guy C, Olauson H, Becker JU, Yang M, Fitzgerald P, et al. ESCRT-III acts downstream of MLKL to regulate necroptotic cell death and its consequences. Cell. 2017;169:286-300. e16.

29. Jeong JH. Inducible mouse models for cancer drug target validation. J Cancer Prev. 2016;21:243-8.

30. Hou W, Han J, Lu C, Goldstein LA, Rabinowich H. Autophagic degradation of active caspase-8: a crosstalk mechanism between autophagy and apoptosis. Autophagy. 2010;6:891-900.

31. Mizushima N, Yoshimori T, Ohsumi Y. The role of Atg proteins in autophagosome formation. Annu Rev Cell Dev Biol. 2011;27:107-32.

32. Lee JA, Beigneux A, Ahmad ST, Young SG, Gao FB. ESCRT-III dysfunction causes autophagosome accumulation and neurodegeneration. Curr Biol. 2007;17:1561-7.

33. Lee JA, Gao FB. Inhibition of autophagy induction delays neuronal cell loss caused by dysfunctional ESCRT-III in frontotemporal dementia. J Neurosci. 2009;29:8506-11.

34. Ghazi-Noori S, Froud KE, Mizielinska S, Powell C, Smidak M, Fernandez de Marco M, et al. Progressive neuronal inclusion formation and axonal degeneration in CHMP2B mutant transgenic mice. Brain. 2012;135:819-32.

35. Rusten TE, Vaccari T, Lindmo K, Rodahl LM, Nezis IP, SemJacobsen C, et al. ESCRTs and Fab1 regulate distinct steps of autophagy. Curr Biol. 2007;17:1817-25.

36. Herz HM, Chen Z, Scherr H, Lackey M, Bolduc C, Bergmann A. vps 25 mosaics display non-autonomous cell survival and overgrowth, and autonomous apoptosis. Development. 2006;133:1871-80.

37. Vaccari T, Bilder D. The Drosophila tumor suppressor vps 25 prevents nonautonomous overproliferation by regulating notch trafficking. Dev Cell. 2005;9:687-98.

38. Thompson BJ, Mathieu J, Sung HH, Loeser E, Rorth P, Cohen SM. Tumor suppressor properties of the ESCRT-II complex component Vps25 in Drosophila. Dev Cell. 2005;9:711-20.

39. Zou W, Liu X, Yue P, Zhou Z, Sporn MB, Lotan R, et al. c-Jun $\mathrm{NH} 2$-terminal kinase-mediated up-regulation of death receptor 5 contributes to induction of apoptosis by the novel synthetic triterpenoid methyl-2-cyano-3,12-dioxooleana-1, 9-dien-28-oate in human lung cancer cells. Cancer Res. 2004;64:7570-8. 
40. Deng Y, Ren X, Yang L, Lin Y, Wu X. A JNK-dependent pathway is required for TNFalpha-induced apoptosis. Cell. 2003;115:61-70.

41. Sharma A, Almasan A. Autophagy as a mechanism of Apo2L/TRAIL resistance. Cancer Biol Ther. 2018;19: 755-62.

42. Lim B, Allen JE, Prabhu VV, Talekar MK, Finnberg NK, ElDeiry WS. Targeting TRAIL in the treatment of cancer: new developments. Expert Opin Ther Targets. 2015;19: 1171-85.

43. Koyama-Honda I, Itakura E, Fujiwara TK, Mizushima N. Temporal analysis of recruitment of mammalian ATG proteins to the autophagosome formation site. Autophagy. 2013;9:1491-9.

44. Tang Z, Takahashi Y, He H, Hattori T, Chen C, Liang X, et al. TOM40 Targets Atg2 to mitochondria-associated ER membranes for phagophore expansion. Cell Rep. 2019;28:1744-57.e45. 